\title{
Stereologic investigation of the posterior part of the hippocampus in schizophrenia
}

\author{
Andrea Schmitt · Corinna Steyskal · Hans-Gert Bernstein • \\ Thomas Schneider-Axmann · Eleni Parlapani - Evelin L. Schaeffer • \\ Wagner F. Gattaz $\cdot$ Bernhard Bogerts $\cdot$ Christoph Schmitz $\cdot$ Peter Falkai
}

Received: 29 March 2008 / Revised: 22 August 2008 / Accepted: 22 August 2008 / Published online: 6 September 2008

(C) The Author(s) 2008

\begin{abstract}
Structural magnetic resonance imaging and postmortem studies showed volume loss in the hippocampus in schizophrenia. The noted tissue reduction in the posterior section suggests that some cellular subfractions within this structure might be reduced in schizophrenia. To address this, we investigated numbers and densities of neurons, oligodendrocytes and astrocytes in the posterior hippocampal subregions in postmortem brains from ten patients with schizophrenia and ten matched controls using design-based stereology performed on Nissl-stained sections. Compared to the controls, the patients with schizo-
\end{abstract}

Electronic supplementary material The online version of this article (doi:10.1007/s00401-008-0430-y contains supplementary material, which is available to authorized users.

A. Schmitt $(\bowtie) \cdot$ T. Schneider-Axmann · E. Parlapani · P. Falkai

Department of Psychiatry, University of Goettingen,

von-Siebold Str. 5, 37075 Göttingen, Germany

e-mail: aschmit@gwdg.de

C. Steyskal

Department of Psychiatry, University of Saarland,

Kirrberger Str, 66421 Homburg/Saar, Germany

H.-G. Bernstein · B. Bogerts

Department of Psychiatry, University of Magdeburg,

Leipziger Str. 44, 39120 Magdeburg, Germany

E. L. Schaeffer - W. F. Gattaz

Department of Psychiatry and Laboratory of Neuroscience

(LIM-27), Faculty of Medicine, University of Sao Paulo,

Rua Dr. Ovidio Pires de Campos s/n, P. O. box 3671,

05403-010 São Paulo, SP, Brazil

C. Schmitz

Division of Neuroscience,

School for Mental Health and Neuroscience,

Maastricht University, P. O. Box 616,

6200 MD Maastricht, Netherlands phrenia showed a significant decrease in the mean number of oligodendrocytes in the left and right CA4. This is the first finding of reduced numbers of oligodendrocytes in CA4 of the posterior part of the hippocampus in schizophrenia. Our results are in line with earlier findings in the literature concerning decreased numbers of oligodendrocytes in the prefrontal cortex in schizophrenia. Our results may indicate disturbed connectivity of the CA4 of the posterior part of the hippocampus in schizophrenia and, thus, contribute to the growing number of studies showing the involvement of posterior hippocampal pathology in the pathophysiology of schizophrenia.

Keywords Hippocampus $\cdot$ Stereology $\cdot$ Cell number · Oligodendrocytes $\cdot$ Schizophrenia

\section{Introduction}

The human hippocampus is involved in memory and regulation of affect, both of which are compromised in schizophrenia [34]. Structural magnetic resonance imaging and postmortem studies in schizophrenia have shown volume loss in the medial temporal region, especially in the hippocampus, as one of the most consistent structural abnormalities [32]. Additionally, postmortem studies showed volume loss in the hippocampal subregions in schizophrenia, which may be related to positive symptoms [12-14]. The small tissue reduction of approximately 5\% suggests that some cellular subfractions within this structure might be reduced in number. However, a recent postmortem study of hippocampal gray matter in schizophrenia using the gray-levelindex (GLI) method revealed no cytoarchitectonic alterations, suggesting no changes in neuronal perikarya [40]. Earlier quantitative studies of the hippocampus described reduced 
density of interneurons, mainly of parvalbumin-immunoreactive cells, without alterations of pyramidal neurons $[9$, 84]. While most of these studies reported no alterations in cell density $[3,20,29]$, the density of pyramidal neurons has been shown to be decreased in schizophrenia [23]. Additionally, there are findings of both smaller [7, 83] or unchanged [35] pyramidal neuron volumes in schizophrenia. Although some authors have noted a reduction of glial cells in the hippocampus of male patients with schizophrenia [23], astrogliosis has not been detected in the hippocampus of postmortem brains from patients with schizophrenia [17, $24,65]$. However, two-dimensional cell counting in only a few sections without considering the volume of the region carries methodological limitations through the influence of volume differences (e.g., mediated by neuropil degeneration) and tissue shrinkage by fixation procedures. Additionally, problems may be caused by irregular cell shape and size, non-random orientation and cutting of cells during sectioning [79]. To date, two design-based stereologic estimates of total cell numbers in the hippocampus reported no differences between patients with schizophrenia and matched controls. It should be noted, however, that one study investigated only the total number of neurons [33], while the second study failed to differentiate between different cell types and included both patients with schizophrenia and schizoaffective patients [75]. Thus, the differentiation of neuronal and glial cell types has not actually been investigated using stereologic methods.

A large number of structural MRI studies has shown a reduced volume of the posterior part of the hippocampus [ 6 , $14,36,51,57,73,82]$, while only some others have found a pronounced reduction in the anterior part [54, 67]. In functional MRI studies, decreased activation of the posterior part of the hippocampus has been related to smooth pursuit eye movement deficits [69] and impaired verbal learning in schizophrenia [22]. Additionally, in the posterior part of the hippocampus, cornu ammonis (CA) subregions can be identified based on histological criteria using Nissl-stained sections [23]. To shed light on alterations in this region, we examined hippocampal subregions (CA1, CA2/3, CA4 and subiculum) in serial postmortem sections from the posterior part of the hippocampus using design-based stereology, posting the hypothesis that the numbers of neurons and oligodendrocytes are decreased in schizophrenia.

\section{Material and methods}

\section{Human postmortem brains}

Postmortem brains were obtained from the Düsseldorf brain collection [13]. Patients fulfilled ICD-9 criteria for schizophrenia. They had been treated with neuroleptic drugs for most of their illness. Exclusion criteria were alcohol or drug abuse and other neuropsychiatric disorders. Brains from ten patients with schizophrenia [mean age $55.1 \pm 7.7$ years (mean \pm SEM), five males, five females, postmortem interval (PMI) $40.0 \pm 17.0 \mathrm{~h}$, mean disease duration $24.8 \pm 7.5$ years] and ten age and gender matched healthy controls without a history of neuropsychiatric disorders, alcohol or drug abuse, dementia, neurological illness, trauma or chronic terminal diseases (mean age $50.2 \pm 10.1$ years, 5 males, 5 females, PMI $41.4 \pm 23.4 \mathrm{~h}$ ) (Table 1) were in toto uniformly fixed in $10 \%$ phosphatebuffered paraformaldehyde for about 7 months $(\mathrm{pH} 7.0$, $\left.T=15-20^{\circ} \mathrm{C}\right)$. Then, the frontal and occipital lobes were separated from the middle part of the brain by coronal sections anterior to the genu of the corpus callosum and posterior to the splenium. These sections contain both hemispheres. The frontal and middle block including the entire hippocampus were embedded in paraffin and cut into $20-\mu \mathrm{m}$ whole brain coronal sections on a Polycut S Leica microtome. Every 50th section (intersectional distance, $1 \mathrm{~mm}$ ) was Nissl (cresyl violet) and myelin (luxol fast-blue) stained. Afterwards, the actual thickness of each section after histological processing was controlled by focussing the upper and lower surface with a light microscope at a magnification of $1,000 \times$. The mean section thickness after histological processing was $18.9 \pm 1.2 \mu \mathrm{m}$.

Within the hippocampal formation we analyzed the posterior part, spanning from the lateral geniculate nucleus to the level of the splenium of the corpus callosum. Subregions to be investigated separately were CA (cornu ammonis) 1, 2-3 and 4 (deep polymorph layer of the dentate gyrus) and subiculum $[55,76]$. According to the literature [21], CA2 and CA3 were lumped together because these small regions are difficult to separate on the microscopic level due to histological criteria. (Note that the anatomical delineation of CA3 and CA4 is a traditional way to segment the hippocampus, but has no functional consequence, since the CA4 neurons originate in CA3 with extensions into the hilus of the dentate gyrus [1]. Thus, according to a new functional view, CA3 and CA4 neurons should be considered together as one hippocampal subregion.) We differentiated between neurons, oligodendroglia and astroglia based on histological and morphological criteria in our Nisslstained sections (Fig. 1). Neurons were distinguished from other cell types on the basis of cytological characteristics. Neurons showed a large cytoplasm, less distinct nuclear membrane, clearly visible nucleolus within a pale nucleus and a less heterogeneous distribution of chromatin material in the nucleus. Oligodendroglial cells were identified by the absence of cytoplasmic staining, intense staining of the nucleus with dispersed chromatin and lack of a nucleolus [72]. Astrocytes were less densely stained and showed a clear rim of cytoplasm [38, 62, 64]. We determined 
Table 1 List of normal controls and patients with schizophrenia investigated in the present study

\begin{tabular}{lllllll}
\hline No. & G & S & A & D & Most prominent symptoms & Cause of death \\
\hline N3 & C & M & 56 & & & Acute pancreatitis \\
N10 & C & M & 50 & & & Myocardial infarction \\
N19 & C & F & 33 & & & Coronary thrombosis \\
N20 & C & M & 64 & & & Rupture of an aneurysm of the abdominal aorta \\
N21 & C & F & 52 & & & Ovarial carcinoma \\
N22 & C & F & 47 & & & Renal failure \\
N23 & C & M & 38 & & & Acute cardiac insufficiency \\
N27 & C & F & 50 & & & Rupture of an aneurysm of the aorta \\
N29 & C & M & 47 & & & Coronary thrombosis \\
N34 & C & F & 64 & & & Peritonitis \\
P3 & P & M & 48 & 18 & Auditory hallucinations, thought disorder & Cardiac failure \\
P7 & P & F & 66 & 30 & Auditory hallucinations, persecution delusions & Myocardial infarction \\
P9 & P & M & 65 & 26 & Persecutions complex, auditory hallucinations & Pulmonary insufficiency \\
P10 & P & M & 46 & 18 & Delusions, paranoid ideas, mutism, negativism & Pulmonary embolism \\
P12 & P & F & 53 & 20 & Auditory and visual hallucinations, delusions, lack of drive & Myocardial infarction \\
P13 & P & F & 60 & 16 & Delusions, mutism, netagivism & Bronchopneumonia \\
P14 & P & M & 51 & 28 & Delusions, mutism, mannerism & Ileus \\
P18 & P & F & 63 & 23 & Auditory hallucinations, persecution, thought disorder & Suicide \\
P20 & P & M & 47 & 23 & Auditory hallucinations, delusions of being poisoned & Cardiac arrest by ventricular fibrillation \\
P23 & P & F & 52 & 28 & Auditory hallucinations, paranoid ideas & Suicide by drowning \\
\hline G & C & & P & &
\end{tabular}

$G$ group, $C$ control, $P$ patient with schizophrenia, $G$ gender, $M$ male, $F$ female, $A$ age (years), $D$ duration of the disease (years)

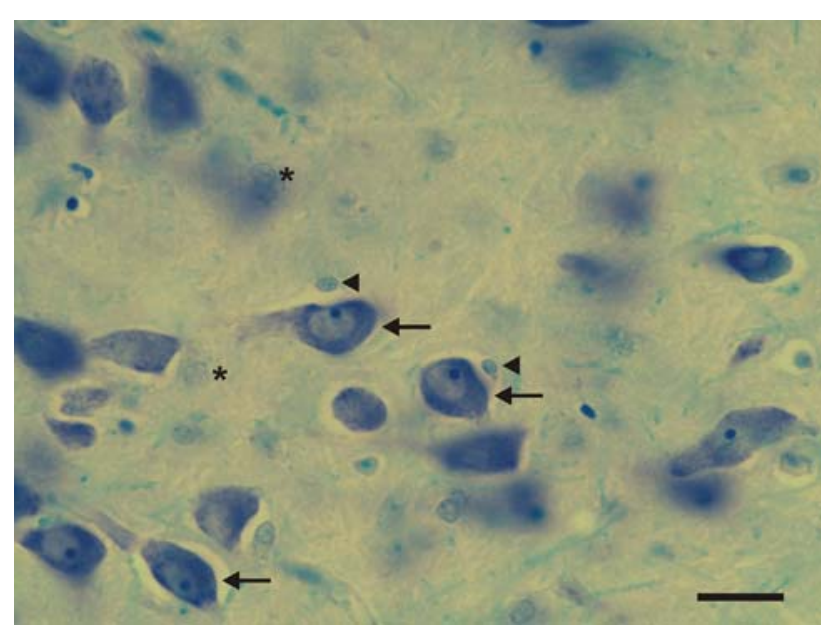

Fig. 1 Representative high-power photomicrograph of a $20 \mu \mathrm{m}$-thick coronal section from a control. The arrows point to neurons, the arrowheads to astrocytes and the asterisks to oligodendrocytes. Scale bar $=25 \mu \mathrm{m}$

densities and numbers of these specific cell types along with the volumes of the entire subregions within the posterior part of the hippocampus.

Stereologic analyses

All stereological analyses were performed with the rater (C.St.) blind to diagnosis using a stereologic workstation, consisting of a modified light microscope (BX50; Olympus, Tokyo, Japan), Olympus Uplan Apo objectives $(1.5 \times$, $20 \times, 50 \times$ oil, $100 \times$ oil), motorized specimen stage for automatic sampling, electronic microcator, CCD color video camera, PC with frame grabber board, stereology software (Stereoinvestigator, MBF Bioscience, Williston, USA) and a 17-in. monitor. Boundaries of hippocampal subfields were traced on video images displayed on the computer screen (Fig. 2) and total volumes were calculated according to Cavalieri's principle [59] using on average $10.1 \pm 2.3$ sections in controls and $10.0 \pm 1.6$ sections in patients with schizophrenia. Total numbers of cells were estimated using the optical fractionator [58-60, 77]. We performed a count of the different cells (neurons, oligodendroglial cells, astrocytes) that came into focus within unbiased virtual counting spaces [26, 66] distributed in a systematic-random fashion throughout the different regions of interest. Estimated cell numbers were calculated from the numbers of counted cells and the sampling probability. The predicted coefficient of error (CE) of the total numbers of neurons, astrocytes and oligodendrocytes was evaluated using the prediction methods described by Schmitz [58] and Schmitz and Hof [60]. Details of the stereologic counting procedure are outlined in Table 2.

The rater was blind to the diagnosis and performed repeated measurements of both the left and right CA2/3 in eight cases. For the intra-rater reliability the intraclass 


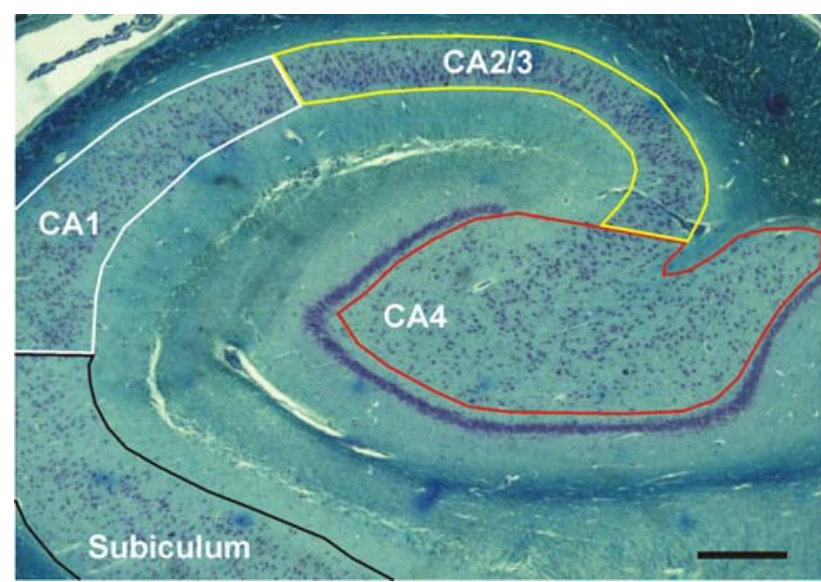

Fig. 2 Representative photomicrograph of a $20 \mu \mathrm{m}$-thick frontal section from a control, showing the subregions of the posterior part of the hippocampus investigated in the present study. Scale bar $=1 \mathrm{~mm}$

correlation coefficients (ICC) were 0.982 for astrocytes, 0.992 for oligodendrocytes, 0.984 for neurons and 1.00 for volumes of the subregions.

\section{Statistical analysis}

For both, patients with schizophrenia and controls, mean and standard error of the mean were calculated for all investigated variables, separately for the left and the right hemispheres. Comparisons between patients with schizophrenia and controls were performed using generalized linear model multivariate analysis (MANOVA), with diagnosis and hemisphere as fixed factors and the following variables as covariates: (i) the patients gender, (ii) the postmortem interval and (iii) the adjusted illness duration of the patients with schizophrenia (calculated as individual age at death minus age at onset plus the mean age at onset of all schizophrenic patients) or the age of the controls, respectively [Note that use of the actual individual illness duration of the patients with schizophrenia instead of the adjusted ones as covariate would have caused invalid results of the MANOVA model because there was no illness duration of the controls, and the mean illness duration of the schizophrenic patients was significantly different from the mean age of the controls (Student's two-tailed $t$ test; $P<0.001$ ), whereas the mean adjusted illness duration was not $(P=0.974)$ ] (see also [46]). For each investigated variable, all investigated brain regions were tested simultaneously. Post hoc tests in the analyses of covariance were performed with linear regression analysis (in case of the adjusted illness duration of the patients with schizophrenia or the age of the controls, respectively), or with two-way repeated measures (RM) ANOVA (in case of the patients gender). In all analyses, an effect was considered statistically significant if its associated $P$ value was smaller than 0.05 . Calculations were performed using SPSS (Version 12.0.1 for Windows, SPSS, Chicago, IL).

\section{Results}

No significant differences between the patients with schizophrenia and the controls were found with respect to the mean volumes, the mean densities of neurons and the mean numbers of neurons in the investigated subregions of the posterior part of the hippocampus (Figs. 3, 4, 5; Fig. S1 in Electronic Supplement Material; Table 3; Table S1 in Electronic Supplement Material show all $P$ values of the MANOVAs performed).

Neither the mean density nor the mean number of astrocytes was significantly different between the patients with schizophrenia and the controls in any of the investigated

Table 2 Details of the stereologic counting procedures

\begin{tabular}{lllll}
\hline & CA1 & CA2/3 & CA4 & Subiculum \\
\hline Obj & $50 \times$ & $50 \times$ & $50 \times$ & $50 \times$ \\
$\mathrm{B}\left(\mu \mathrm{m}^{2}\right)$ & 900 & 900 & 900 & 900 \\
$\mathrm{H}(\mu \mathrm{m})$ & 17 & 17 & 17 & 17 \\
$\mathrm{D}(\mu \mathrm{m})$ & $170 \times 340$ & $455 \times 170$ & $430 \times 290$ & $1,050 \times 400$ \\
$\sum \mathrm{CS}$ & 150 & 150 & 200 & 400 \\
$\sum \mathrm{Q}^{-}$neurons & 834 & 844 & 1607 & 1518 \\
$\sum \mathrm{Q}^{-}$astrocytes & 182 & 253 & 390 & 368 \\
$\sum \mathrm{Q}^{-}$oligodendrocytes & 367 & 377 & 843 & 803 \\
$\mathrm{CE} E_{\text {pred }}$ (neurons) & 0.038 & 0.047 & 0.038 & 0.040 \\
$\mathrm{CE}_{\text {pred }}$ (astrocytes) & 0.051 & 0.053 & 0.048 & 0.046 \\
$\mathrm{CE}_{\text {pred }}$ (oligodendrocytes $)$ & 0.036 & 0.041 & 0.037 & 0.042 \\
\hline
\end{tabular}

Obj objective used, $B$ and $H$ base and height of the unbiased virtual counting spaces, $D$ distances between the unbiased virtual counting spaces in mutually orthogonal directions $\mathrm{x}$ and y, $\Sigma C S$ average sum of unbiased virtual counting spaces used in one hemisphere, $\Sigma Q^{-}$average number of counted neurons, astrocytes and oligodendrocytes in one hemisphere; $C E_{\text {pred }}$ average predicted coefficient of error of the estimated cell numbers 
Fig. 3 Representative highpower photomicrographs of $20 \mu \mathrm{m}$-thick coronal sections of the dorsal part of the hippocampus of a patients with schizophrenia (a, c, e) and a control (b, d, f), showing details of the subregions CA4 $(\mathbf{a}, \mathbf{b})$, $\mathrm{CA} 2 / 3(\mathbf{c}, \mathbf{d})$ and CA1 $(\mathbf{e}, \mathbf{f})$. Note that the reduced number of oligodendrocytes in CA4 in schizophrenia was not necessarily obvious at simple observation of the tissue but necessitate the type of rigorous quantitative analysis undertaken in this study to be revealed. Scale bar $=50 \mu \mathrm{m}$
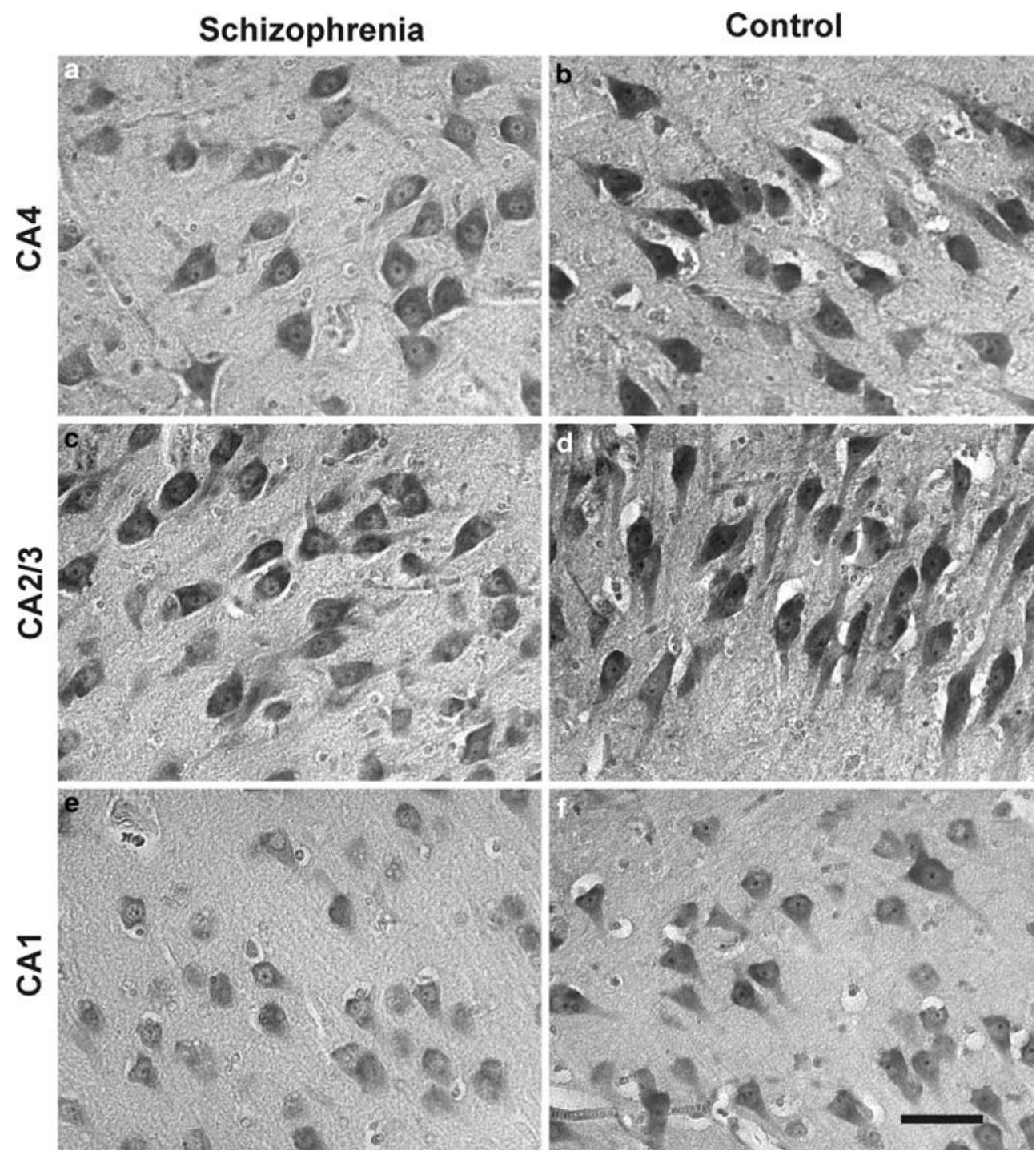

subregions of the posterior part of the hippocampus (Fig. 6; Fig. S2 in Electronic Supplement Material, respectively). However, multivariate analysis revealed an effect of gender $(F=5.088$; degree of freedom $=4.0$ [this was the case in all MANOVAs applied]; $P=0.03$ ) and age (controls) or duration of the disease (patients) on the number of astrocytes $(F=13.621 ; P<0.001)$ as well an effect of age (controls) or duration of the disease (patients) on the density of astrocytes $(F=9.014 ; P<0.001)$. Additionally, we found a significant, positive relation between (i) the density of astrocytes and the controls age in CA4 and the subiculum, (ii) the density of astrocytes and the age of the patients with schizophrenia in CA1 and the subiculum, (iii) the number of astrocytes and the controls age in CA2/3, CA4 and the subiculum, and (iv) the number of astrocytes and the age of the patients with schizophrenia in CA1 and the subiculum, respectively (Fig. 6; Fig. S2 in Electronic Supplement Material, respectively).
MANOVA also showed significant effects of diagnosis $(F=3.587 ; P=0.017)$ and gender $(F=3.841 ; P=0.012)$ on the mean numbers of oligodendrocytes. Subsequent univariate tests demonstrated then that, compared to the controls, the patients with schizophrenia showed a significantly reduced mean number of oligodendrocytes only in CA4 $[-27.6 \%$ on the left side (l) and $-37.8 \%$ on the right side (r); $F_{(1)}=6.767$ (one degree of freedom); $P=0.014$ for the fixed factor Diagnosis in the MANOVA] (Fig. 7; Fig. S3 in Electronic Supplement Material). For CA1, CA2/3 and the subiculum, no significant differences in the mean number of oligodendrocytes were found between the patients with schizophrenia and the controls. Note that the reduced number of oligodendrocytes in CA4 in schizophrenia was not necessarily obvious at simple observation of the tissue (as shown in Fig. 3), but necessitate the type of rigorous quantitative analysis undertaken in this study to be revealed. 
Fig. 4 Volumes of CA1 (a-c), CA2/3 (d-f), CA4 (g-i) and the subiculum $(\mathbf{k}-\mathbf{m})$ in both hemispheres of the brains from ten patients with schizophrenia ( $S$ open bars in $\mathbf{a}, \mathbf{d}, \mathbf{g}, \mathbf{k}$, and dots and squares in $\mathbf{c}, \mathbf{f}, \mathbf{i}, \mathbf{m})$ and ten age-matched controls

(C; closed bars in $\mathbf{a}, \mathbf{d}, \mathbf{g}, \mathbf{k}$, and dots and squares in $\mathbf{b}, \mathbf{e}, \mathbf{h}, \mathbf{l})$. In $\mathbf{a}, \mathbf{d}, \mathbf{g}, \mathbf{k}$, data are shown as mean and standard error of the mean for the left (l) and right (r) hemispheres from patients with schizophrenia ( $S$ - $l$ and $S-r$ ) and controls $(C-l$ and $C-r)$. In $\mathbf{b}, \mathbf{c}, \mathbf{e}$, $\mathbf{f}, \mathbf{h}, \mathbf{i}, \mathbf{l}, \mathbf{m}$, individual data for the left hemispheres (closed dots males, open dots females) and right hemispheres (closed squares males, open squares females) from controls $(\mathbf{b}, \mathbf{e}, \mathbf{h}, \mathbf{l})$ and patients with schizophrenia $(\mathbf{c}, \mathbf{f}, \mathbf{i}, \mathbf{m})$ are shown as function of the controls age (or the illness duration of the patients with schizophrenia, respectively)
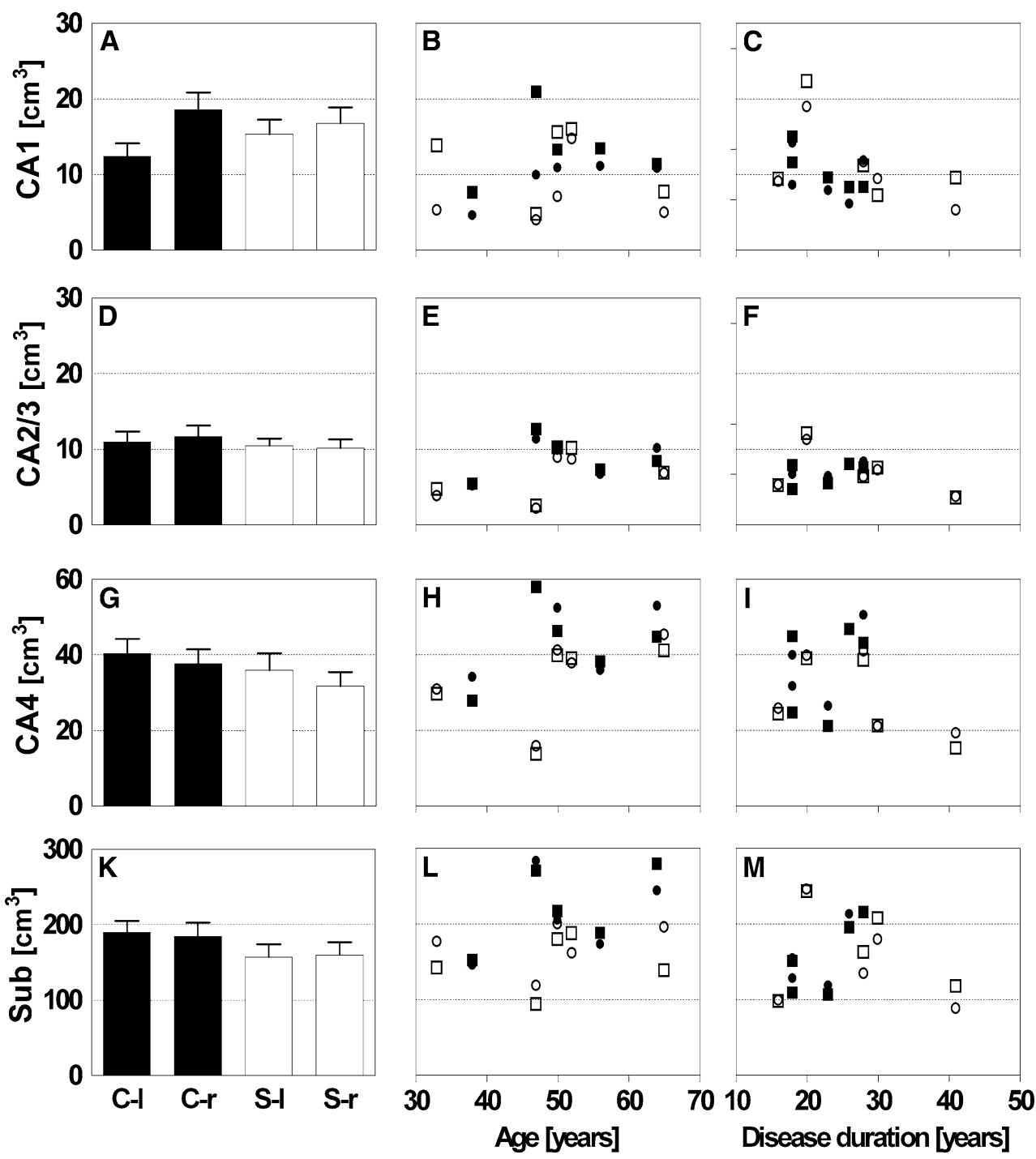

The postmortem interval and the hemispheres had not any influence on the investigated variables. In contrast, MANOVA showed a significant effect of gender on the mean volumes of the subregions of the posterior part of the hippocampus $(F=2.930 ; P=0.037)$, as well as on the mean numbers of neurons within these subregions $(F=3.295 ; \quad P=0.024)$. Subsequent univariate analysis showed that the gender of the controls and the patients with schizophrenia had a significant influence on the volume of CA4 as well as the number of neurons in CA2/3, CA4 and the subiculum. By pooling the data from the controls and the patients with schizophrenia, we found that on average the females had a significantly smaller volume of CA4 than the males $\left[-28.9 \%\right.$ (1) and $-23.8 \%(\mathrm{r}) ; P_{\text {hemisphere }}=0.097$; $\left.P_{\text {gender }}=0.019 ; P_{\text {interaction }}=0.148\right]$ as well as significantly smaller numbers of neurons in CA2/3 $[-30.9 \%$ (l) and $-16.8 \% \quad(\mathrm{r}) ; \quad P_{\text {hemisphere }}=0.389 ; \quad P_{\text {gender }}=0.044$; $\left.P_{\text {interaction }}=0.019\right]$ and CA4 [-29.5\% (l) and $-23.7 \%(\mathrm{r})$; $\left.P_{\text {hemisphere }}=0.132 ; P_{\text {gender }}=0.035 ; P_{\text {interaction }}=0.154\right]$ but not in the subiculum ${ }_{\text {Hemisphere }}=0.892 ; P_{\text {gender }}=0.068$; $\left.P_{\text {interaction }}=0.654\right) \quad$ (Fig. S4 in Electronic Supplement Material).

\section{Discussion}

The present postmortem study revealed no differences in mean neuron numbers or neuron densities in the subfields of the posterior part of the hippocampus between patients with schizophrenia and controls. In line with our results, Heckers et al. [33] and Walker et al. [75] also did not detect differences in neuronal numbers in all cornu ammonis subdivisions of the total hippocampus in schizophrenia using a stereologic approach. Thus, we could not confirm earlier findings of a decreased density of pyramidal cells in the hippocampus in schizophrenia [23]. In contrast, Benes et al. [10] found reduced numbers of pyramidal neurons in CA1 of the posterior part of the hippocampus in patients with 
Fig. 5 Number of neurons in CA1 (a-c), CA2/3 (d-f), CA4 $(\mathbf{g}-\mathbf{i})$ and the subiculum $(\mathbf{k}-\mathbf{m})$ in both hemispheres of the brains from ten patients with schizophrenia ( $\mathbf{S}$; open bars in $\mathbf{a}, \mathbf{d}, \mathbf{g}$, $\mathbf{k}$, and dots and squares in $\mathbf{c}, \mathbf{f}, \mathbf{i}$, m) and ten age-matched controls (C; closed bars in $\mathbf{a}, \mathbf{d}, \mathbf{g}, \mathbf{k}$, and dots and squares in $\mathbf{b}, \mathbf{e}, \mathbf{h}, \mathbf{l})$. In $\mathbf{a}, \mathbf{d}, \mathbf{g}, \mathbf{k}$, data are shown as mean and standard error of the mean for the left (l) and right (r) hemispheres from patients with schizophrenia ( $S$ - $l$ and $S-r$ ) and controls $(C-l$ and $C-r)$. In $\mathbf{b}, \mathbf{c}, \mathbf{e}$, $\mathbf{f}, \mathbf{h}, \mathbf{i}, \mathbf{l}, \mathbf{m}$, individual data for the left hemispheres (closed dots males, open dots females) and right hemispheres (closed squares males, open squares females) from controls (b, e, h, l) and patients with schizophrenia $(\mathbf{c}, \mathbf{f}, \mathbf{i}, \mathbf{m})$ are shown as function of the controls age (or the illness duration of the patients with schizophrenia, respectively
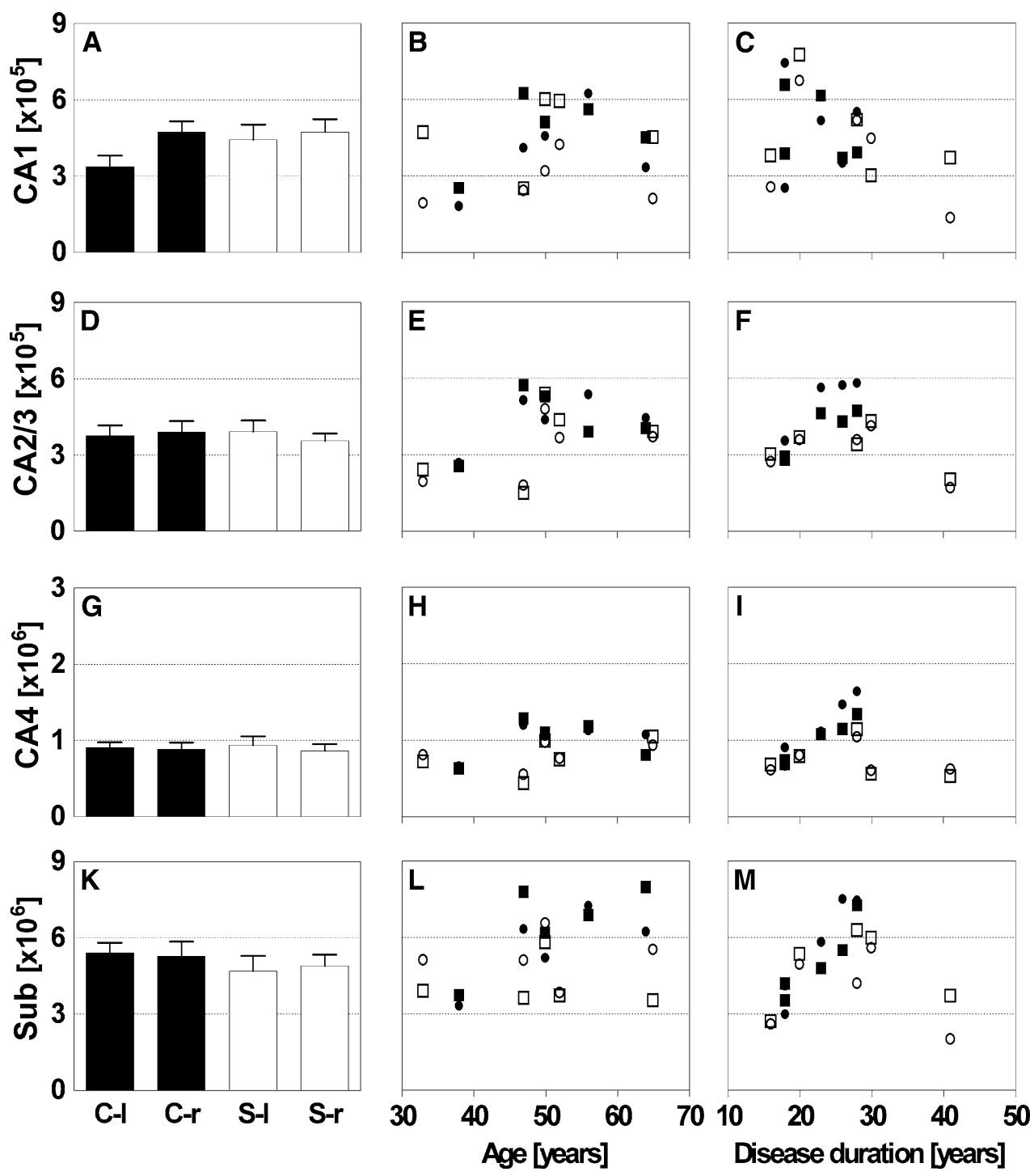

schizophrenia which also suffered from mood disturbances. Since we did not investigate a group of patients with affective disorders, it cannot be excluded that neuron number is altered in patients with mood disturbances. Additionally, we did not distinguish between pyramidal neurons and interneurons, because these cell types cannot be separated in Nissl-stained sections due to overlapping morphological criteria.

We detected effects of age on density and number of astrocytes in several subregions, but no effects of diagnosis on astrocyte number. Our findings confirm animal studies showing increased mean astrocyte numbers in brains of aged mice [50], and the well-known lack of astrocytosis in schizophrenia [17, 24]. Thus, the increasing number of astrocytes in the ageing brain shown in our study might serve as internal validity control for the results from the stereologic investigations. Furthermore, we did not find effects of age on neuronal number in our study. Another study showed that age had only little influence on this parameter in the human brain [53]. However, we did not confirm a decline in the number of neurons in the hilus and subiculum of the hippocampus, which has been shown by West and colleagues [32]. Altogether, age-related effects on neurons may reflect neuronal dysfunction rather than cell loss [49].

According to our results of fewer neurons in CA4 and CA2/3 in the brains from females compared to males, male gender is known to be related to larger neuron numbers in the subiculum of the rat hippocampus [2], although interspecies differences may render comparisons between rats and humans difficult. While a stereologic study in human cortical regions did show a higher neuronal number in men [63], these results may not be related to changes in the hippocampus. Since the size of our samples of males and females was small, our results should be taken with caution in this regard and confirmed in a larger cohort.

We found a decrease in the mean number of oligodendrocytes in the left and right CA4 of the posterior part of the hippocampus in schizophrenia compared to controls. 
Table 3 Results of statistical analysis ( $P$ values) with generalized linear model multivariate analysis of variance (MANOVA)

\begin{tabular}{|c|c|c|c|c|c|c|c|}
\hline Variable & Subregion & $\mathrm{A} / \mathrm{ID}$ & PMI & S & $\mathrm{D}$ & $\mathrm{H}$ & $\mathrm{D} \times \mathrm{H}$ \\
\hline \multirow{5}{*}{ Volume } & All subregions & 0.321 & 0.974 & $\mathbf{0 . 0 3 7}$ & 0.154 & 0.141 & 0.696 \\
\hline & CA1 & & & 0.822 & & & \\
\hline & $\mathrm{CA} 2 / 3$ & & & 0.141 & & & \\
\hline & CA4 & & & 0.003 & & & \\
\hline & Subiculum & & & 0.059 & & & \\
\hline \multirow[t]{5}{*}{ Number of neurons } & All subregions & 0.237 & 0.813 & 0.024 & 0.246 & 0.292 & 0.712 \\
\hline & CA1 & & & 0.407 & & & \\
\hline & $\mathrm{CA} 2 / 3$ & & & 0.006 & & & \\
\hline & $\mathrm{CA} 4$ & & & 0.001 & & & \\
\hline & Subiculum & & & 0.010 & & & \\
\hline \multirow[t]{5}{*}{ Number of astrocytes } & All subregions & $<0.001$ & 0.814 & 0.003 & 0.104 & 0.962 & 0.542 \\
\hline & CA1 & 0.014 & & 0.543 & & & \\
\hline & $\mathrm{CA} 2 / 3$ & 0.017 & & 0.246 & & & \\
\hline & CA4 & $<0.001$ & & 0.001 & & & \\
\hline & Subiculum & $<0.001$ & & 0.407 & & & \\
\hline \multirow{5}{*}{$\begin{array}{l}\text { Number of } \\
\text { oligodendrocytes }\end{array}$} & All subregions & 0.821 & 0.782 & 0.012 & 0.017 & 0.688 & 0.982 \\
\hline & CA1 & & & 0.184 & 0.812 & & \\
\hline & $\mathrm{CA} 2 / 3$ & & & 0.396 & 0.171 & & \\
\hline & CA4 & & & 0.283 & 0.014 & & \\
\hline & Subiculum & & & 0.704 & 0.089 & & \\
\hline
\end{tabular}

One of the most pronounced decreases in expression of oligodendrocyte-related proteins have been reported in the hippocampus of patients with schizophrenia [19, 42]. These microarray data and our light microscopic findings in the posterior part of the hippocampus are supported by in vivo diffusion tensor imaging (DTI) studies of white matter tracts of the fornix body and posterior part of the hippocampus in schizophrenia, showing decreased fractional anisotropy and supporting the hypothesis of functional dysconnectivity in schizophrenia [41, 47, 78, 85]. Moreover, cognitive function such as verbal declarative memory is correlated with the fractional anisotropy of the hippocampus in schizophrenia [48]. This disruption of connectivity in this region could result in cognitive as well as clinical negative symptoms $[28,47,56]$. This is due to the fact that neuronal fibers traversing the limbic pathways from the posterior part of the hippocampus are connected to prefrontal regions and pathways involved in higher cognition [25]. The prefrontal cortex and posterior part of the hippocampus belong to a neuronal network showing disturbances in schizophrenia, most likely of neurodevelopmental origin [15].

One major limitation of our study is the partial sampling strategy investigating only the posterior part of the hippocampus, while the very posterior tail of the hippocampus was not always completely included. Additionally, the posterior part of the hippocampus has been defined using external landmarks. The definition, therefore, may vary slightly depending on slight differences in the angle of the cut 
Fig. 6 Number of astrocytes in CA1 (a-c), CA2/3 (d-f), CA4 $(\mathbf{g}-\mathbf{i})$ and the subiculum $(\mathbf{k}-\mathbf{m})$ in both hemispheres of the brains from ten patients with schizophrenia ( $\mathrm{S}$; open bars in $\mathbf{a}, \mathbf{d}, \mathbf{g}$, $\mathbf{k}$, and dots and squares in $\mathbf{c}, \mathbf{f}, \mathbf{i}$, m) and ten age-matched controls (C; closed bars in $\mathbf{a}, \mathbf{d}, \mathbf{g}, \mathbf{k}$, and dots and squares in $\mathbf{b}, \mathbf{e}, \mathbf{h}, \mathbf{l}$ ). In $\mathbf{a}, \mathbf{d}, \mathbf{g}, \mathbf{k}$, data are shown as mean and standard error of the mean for the left (l) and right (r) hemispheres from patients with schizophrenia $(S-l$ and $S$ - $r$ ) and controls $(C-l$ and $C-r)$. In $\mathbf{b}, \mathbf{c}, \mathbf{e}$, $\mathbf{f}, \mathbf{h}, \mathbf{i}, \mathbf{l}, \mathbf{m}$, individual data for the left hemispheres (closed dots males, open dots females) and right hemispheres (closed squares males, open squares females) from controls (b, e, h, l) and patients with schizophrenia $(\mathbf{c}, \mathbf{f}, \mathbf{i}, \mathbf{m})$ are shown as function of the controls age (or the illness duration of the patients with schizophrenia, respectively). In $\mathbf{c}, \mathbf{e}, \mathbf{h}, \mathbf{l}, \mathbf{m}$, results of linear regression analysis (regression lines, $95 \%$ confidence intervals of the regression lines, regression coefficients and corresponding $P$ values, respectively) are shown, indicating statistically significant relations between the number of the astrocytes and the controls age (or the illness duration of the patients with schizophrenia, respectively)
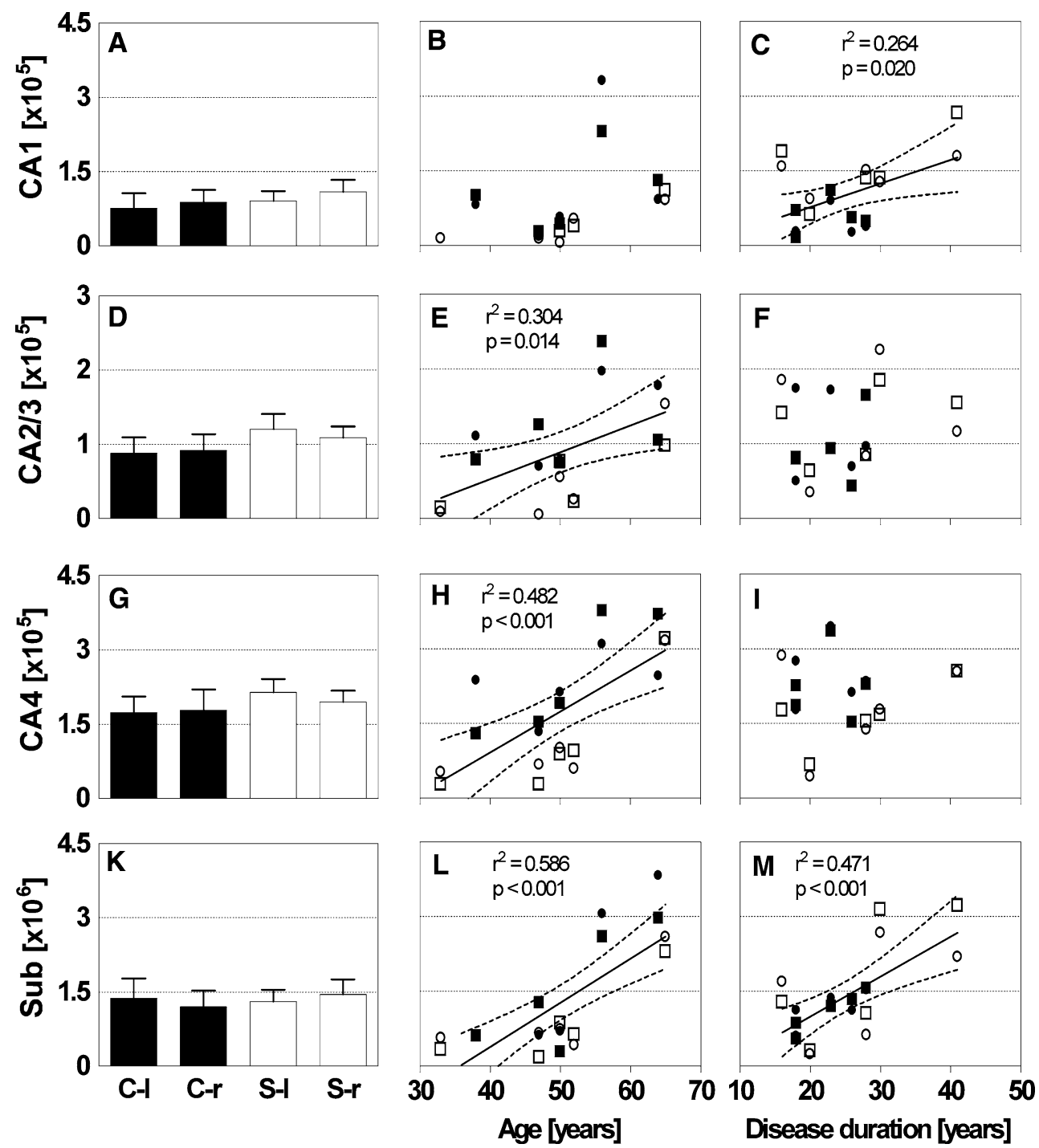

surfaces as well as differences in the volumes of the landmarks. However, meta-analyses of regional brain volumes did not find reductions in the volume of the lateral geniculate nucleus in schizophrenia. The corpus callosum was reduced in volume mainly in first-episode patients, while medicated patients with chronic schizophrenia showed larger volumes [4, 21, 39, 80]. Another limitation is the treatment of patients with neuroleptics, which may influence cell numbers and volumes of hippocampal subregions. In animal studies, antipsychotics reduced astrocytes and, to a non-significant degree, also oligodendrocytes, while increasing neuronal density [44, 45]. In the hippocampus, cell proliferation has been reported to be increased after treatment with atypical antipsychotics [27, 43, 52]. In our study, however, the decreased number of oligodendrocytes in CA4 in the posterior part of the hippocampus of patients with schizophrenia was not related to either the duration of the disease and, thus, the probable duration of medication.
Further studies on animal models are needed to investigate the influence of long-term antipsychotic medication on cell number in the posterior part of the hippocampus.

While it should be noted that the postmortem intervals in our study sample were rather long, we did not find any influence of the postmortem interval on any of the variables investigated. Accordingly, we opted not to exclude cases with longer postmortem intervals.

In summary, we found decreased mean numbers of oligodendrocytes in the left and right posterior hippocampal subfield CA4 in schizophrenia using a design-based stereologic approach. Our results point to disturbances in connectivity of subregions of the posterior part of the hippocampus and confirm earlier findings in the literature of hippocampal pathology in schizophrenia. Further studies investigating the total hippocampus in larger samples and immunohistochemical approaches are called for to replicate these results. Furthermore, animal studies should be 
Fig. 7 Number of oligodendrocytes in CA1 (a-c), CA2/3 (d-f), CA4 $(\mathbf{g}-\mathbf{i})$ and the subiculum $(\mathbf{k}-\mathbf{m})$ in both hemispheres of the brains from ten patients with schizophrenia ( $\mathrm{S}$; open bars in $\mathbf{a}$, $\mathbf{d}, \mathbf{g}, \mathbf{k}$, and dots and squares in $\mathbf{c}, \mathbf{f}, \mathbf{i}, \mathbf{m})$ and ten age-matched controls $(\mathrm{C}$; closed bars in $\mathbf{a}, \mathbf{d}$, $\mathbf{g}, \mathbf{k}$, and dots and squares in $\mathbf{b}$, $\mathbf{e}, \mathbf{h}, \mathbf{l})$. In $\mathbf{a}, \mathbf{d}, \mathbf{g}, \mathbf{k}$, data are shown as mean and standard error of the mean for the left (l) and right ( $\mathrm{r}$ ) hemispheres from patients with schizophrenia $(S-l$ and $S-r)$ and controls $(C-l$ and $C-r)$. In $\mathbf{b}, \mathbf{c}, \mathbf{e}, \mathbf{f}, \mathbf{h}, \mathbf{i}, \mathbf{l}, \mathbf{m}$, individual data for the left hemispheres (closed dots males, open dots females) and right hemispheres (closed squares males, open squares females) from controls (b, e, h, l) and patients with schizophrenia $(\mathbf{c}, \mathbf{f}, \mathbf{i}, \mathbf{m})$ are shown as function of the controls age (or the illness duration of the patients with schizophrenia, respectively). $* P<0.05$ for the fixed factor Diagnosis in general linear model multivariate analysis of variance (MANOVA)
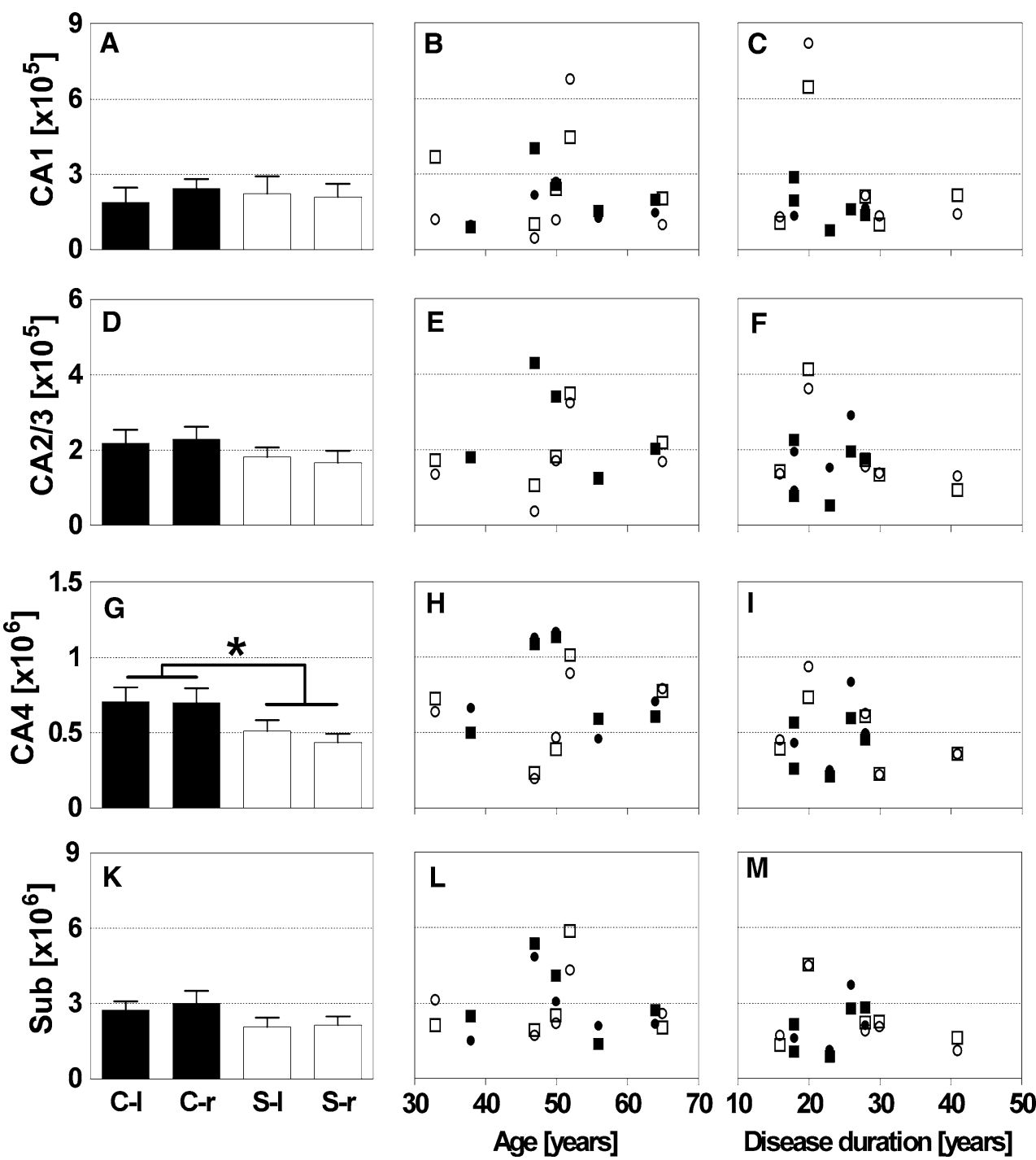

conducted to investigate effects of antipsychotic treatment in more detail.

Acknowledgments This study was supported by the European Commission under the Sixth Framework Programme (BrainNet Europe II, LSHM-CT-2004-503039). The paper reflects only the authors views and the Community is not liable for any use that may be made of it. The authors wish to thank Ms. Waltraud VanSyckel for her valuable input regarding language revision.

Open Access This article is distributed under the terms of the Creative Commons Attribution Noncommercial License which permits any noncommercial use, distribution, and reproduction in any medium, provided the original author(s) and source are credited.

\section{References}

1. Amaral D, Lavanex P (2007) Hippocampal neuroanatomy. In: Andersen P, Morris R, Amaral D, Bliss T, O'Keefe J (eds) The hippocampus book. Oxford University Press, Oxford, pp 37-114
2. Andrade JP, Madeira MD, Paula-Barbosa MM (2000) Sexual dimorphism in the subiculum of the rat hippocampal formation. Brain Res 875:125-137. doi:10.1016/S0006-8993(00)02605-6

3. Arnold SE, Franz BR, Gur RC et al (1995) Smaller neuron size in schizophrenia in hippocampal subfields that mediate corticalhippocampal interactions. Am J Psychiatry 152:738-748

4. Arnone D, McIntosh AM, Tan GM et al (2008) Meta-analysis of magnetic resonance imaging studies of the corpus callosum in schizophrenia. Schizophr Res 101:124-132. doi:10.1016/j.schres. 2008.01.005

5. Bartzokis G, Nuechterlein KH, Lu PH et al (2003) Dysregulated brain development in adult men with schizophrenia: a magnetic resonance imaging study. Biol Psychiatry 53:412-421. doi:10. 1016/S0006-3223(02)01835-8

6. Becker T, Elmer K, Schneider F et al (1996) Confirmation of reduced temporal limbic structure volume on magnetic resonance imaging in male patients with schizophrenia. Psychiatry Res 67:135-143. doi:10.1016/0925-4927(96)03002-8

7. Benes FM (1991) Evidence for neurodevelopment disturbances in anterior cingulate cortex of post-mortem schizophrenic brain. Schizophr Res 5:187-188. doi:10.1016/0920-9964(91)90063-W

8. Benes FM (1989) Myelination of cortical-hippocampal relays during late adolescence. Schizophr Bull 15:585-593 
9. Benes FM, Kwok EW, Vincent SL et al (1998) A reduction of nonpyramidal cells in sector CA2 of schizophrenics and manic depressives. Biol Psychiatry 44:88-97. doi:10.1016/S0006-3223(98) 00138-3

10. Benes FM, Sorensen I, Bird ED (1991) Reduced neuronal size in posterior hippocampus of schizophrenic patients. Schizophr Bull 17:597-608

11. Benes FM, Turtle M, Khan Y et al (1994) Myelination of a key relay zone in the hippocampal formation occurs in the human brain during childhood, adolescence, and adulthood. Arch Gen Psychiatry 51:477-484

12. Bogerts B (1997) The temporolimbic system theory of positive schizophrenic symptoms. Schizophr Bull 23:423-435

13. Bogerts B, Falkai P, Haupts M et al (1990) Post-mortem volume measurements of limbic system and basal ganglia structures in chronic schizophrenics. Initial results from a new brain collection. Schizophr Res 3:295-301. doi:10.1016/0920-9964(90)90013-W

14. Bogerts B, Lieberman JA, Ashtari M et al (1993) Hippocampusamygdala volumes and psychopathology in chronic schizophrenia. Biol Psychiatry 33:236-246. doi:10.1016/0006-3223(93)90289-P

15. Bullmore ET, Frangou S, Murray RM (1997) The dysplastic net hypothesis: an integration of developmental and dysconnectivity theories of schizophrenia. Schizophr Res 28:143-156. doi:10. 1016/S0920-9964(97)00114-X

16. Byne W, Kidkardnee S, Tatusov A et al (2006) Schizophreniaassociated reduction of neuronal and oligodendrocyte numbers in the anterior principal thalamic nucleus. Schizophr Res 85:245253. doi:10.1016/j.schres.2006.03.029

17. Casanova MF, Stevens JR, Kleinman JE (1990) Astrocytosis in the molecular layer of the dentate gyrus: a study in Alzheimer's disease and schizophrenia. Psychiatry Res 35:149-166. doi:10.1016/ 0165-1781(90)90157-Z

18. Corfas G, Roy K, Buxbaum JD (2004) Neuregulin 1-erbB signaling and the molecular/cellular basis of schizophrenia. Nat Neurosci 7:575-580. doi:10.1038/nn1258

19. Dracheva S, Davis KL, Chin B et al (2006) Myelin-associated mRNA and protein expression deficits in the anterior cingulate cortex and hippocampus in elderly schizophrenia patients. Neurobiol Dis 21:531-540. doi:10.1016/j.nbd.2005.08.012

20. Dwork AJ (1997) Postmortem studies of the hippocampal formation in schizophrenia. Schizophr Bull 23:385-402

21. Ellison-Wright I, Glahn DC, Laird AR et al (2008) The anatomy of first-episode and chronic schizophrenia: an anatomical likelihood estimation meta-analysis. Am J Psychiatry 165:1015-1023. doi:10.1176/appi.ajp.2008.07101562

22. Eyler LT, Jeste DV, Brown GG (2008) Brain response abnormalities during verbal learning among patients with schizophrenia. Psychiatry Res 162:11-25. doi:10.1016/j.pscychresns. 2007.03. 009

23. Falkai P, Bogerts B (1986) Cell loss in the hippocampus of schizophrenics. Eur Arch Psychiatry Neurol Sci 236:154-161. doi:10. 1007/BF00380943

24. Falkai P, Honer WG, David S et al (1999) No evidence for astrogliosis in brains of schizophrenic patients. A post-mortem study. Neuropathol Appl Neurobiol 25:48-53. doi:10.1046/j.13652990.1999.00162.x

25. Goldman-Rakic PS, Selemon LD, Schwartz ML (1984) Dual pathways connecting the dorsolateral prefrontal cortex with the hippocampal formation and parahippocampal cortex in the rhesus monkey. Neuroscience 12:719-743. doi:10.1016/0306-4522 (84)90166-0

26. Gundersen HJ, Bagger P, Bendtsen TF et al (1988) The new stereological tools: disector, fractionator, nucleator and point sampled intercepts and their use in pathological research and diagnosis. APMIS 96:857-881

27. Halim ND, Weickert CS, McClintock BW et al (2004) Effects of chronic haloperidol and clozapine treatment on neurogenesis in the adult rat hippocampus. Neuropsychopharmacology 29:10631069. doi:10.1038/sj.npp.1300422

28. Harrison PJ (2004) The hippocampus in schizophrenia: a review of the neuropathological evidence and its pathophysiological implications. Psychopharmacology (Berl) 174:151-162. doi:10.1007/ s00213-003-1761-y

29. Harrison PJ (1999) The neuropathology of schizophrenia. A critical review of the data and their interpretation. Brain 122(Pt 4):593-624. doi:10.1093/brain/122.4.593

30. Harrison PJ, Eastwood SL (2001) Neuropathological studies of synaptic connectivity in the hippocampal formation in schizophrenia. Hippocampus 11:508-519. doi:10.1002/hipo.1067

31. Harrison PJ, Weinberger DR (2005) Schizophrenia genes, gene expression, and neuropathology: on the matter of their convergence. Mol Psychiatry 10:40-68 image 45

32. Heckers S (2001) Neuroimaging studies of the hippocampus in schizophrenia. Hippocampus 11:520-528. doi:10.1002/hipo.1068

33. Heckers S, Heinsen H, Geiger B et al (1991) Hippocampal neuron number in schizophrenia. A stereological study. Arch Gen Psychiatry 48:1002-1008

34. Heckers S, Konradi C (2002) Hippocampal neurons in schizophrenia. J Neural Transm 109:891-905. doi:10.1007/s007020200073

35. Highley JR, Walker MA, McDonald B et al (2003) Size of hippocampal pyramidal neurons in schizophrenia. Br J Psychiatry 183:414-417. doi:10.1192/bjp.183.5.414

36. Hirayasu Y, Shenton ME, Salisbury DF et al (1998) Lower left temporal lobe MRI volumes in patients with first-episode schizophrenia compared with psychotic patients with first-episode affective disorder and normal subjects. Am J Psychiatry 155:13841391

37. Hof PR, Haroutunian V, Copland C et al (2002) Molecular and cellular evidence for an oligodendrocyte abnormality in schizophrenia. Neurochem Res 27:1193-1200. doi:10.1023/A:1020 981510759

38. Hof PR, Haroutunian V, Friedrich VL Jr et al (2003) Loss and altered spatial distribution of oligodendrocytes in the superior frontal gyrus in schizophrenia. Biol Psychiatry 53:1075-1085. doi:10.1016/S0006-3223(03)00237-3

39. Honea R, Crow TJ, Passingham D et al (2005) Regional deficits in brain volume in schizophrenia: a meta-analysis of voxel-based morphometry studies. Am J Psychiatry 162:2233-2245. doi:10. 1176/appi.ajp.162.12.2233

40. Hurlemann R, Tepest R, Maier W et al (2005) Intact hippocampal gray matter in schizophrenia as revealed by automatized image analysis postmortem. Anat Embryol (Berl) 210:513-517. doi:10.1007/s00429-005-0035-3

41. Kalus P, Buri C, Slotboom J et al (2004) Volumetry and diffusion tensor imaging of hippocampal subregions in schizophrenia. Neuroreport 15:867-871. doi:10.1097/00001756-20040409000027

42. Katsel P, Davis KL, Haroutunian V (2005) Variations in myelin and oligodendrocyte-related gene expression across multiple brain regions in schizophrenia: a gene ontology study. Schizophr Res 79:157-173. doi:10.1016/j.schres.2005.06.007

43. Kodama M, Fujioka T, Duman RS (2004) Chronic olanzapine or fluoxetine administration increases cell proliferation in hippocampus and prefrontal cortex of adult rat. Biol Psychiatry 56:570-580. doi:10.1016/j.biopsych.2004.07.008

44. Konopaske GT, Dorph-Petersen KA, Pierri JN et al (2007) Effect of chronic exposure to antipsychotic medication on cell numbers in the parietal cortex of macaque monkeys. Neuropsychopharmacology 32:1216-1223. doi:10.1038/sj.npp.1301233

45. Konopaske GT, Dorph-Petersen KA, Sweet RA et al (2008) Effect of chronic antipsychotic exposure on astrocyte and oligodendrocyte numbers in macaque monkeys. Biol Psychiatry 63:759-765. doi:10.1016/j.biopsych.2007.08.018 
46. Kreczmanski P, Heinsen H, Mantua V et al (2007) Volume, neuron density and total neuron number in five subcortical regions in schizophrenia. Brain 130:678-692. doi:10.1093/brain/awl386

47. Kuroki N, Kubicki M, Nestor PG et al (2006) Fornix integrity and hippocampal volume in male schizophrenic patients. Biol Psychiatry 60:22-31. doi:10.1016/j.biopsych.2005.09.021

48. Lim KO, Ardekani BA, Nierenberg J et al (2006) Voxelwise correlational analyses of white matter integrity in multiple cognitive domains in schizophrenia. Am J Psychiatry 163:2008-2010. doi:10.1176/appi.ajp.163.11.2008

49. Long JM, Mouton PR, Jucker M et al (1999) What counts in brain aging? Design-based stereological analysis of cell number. J Gerontol 54:B407-B417

50. Mouton PR, Long JM, Lei DL et al (2002) Age and gender effects on microglia and astrocyte numbers in brains of mice. Brain Res 956:30-35. doi:10.1016/S0006-8993(02)03475-3

51. Narr KL, Thompson PM, Sharma T et al (2001) Three-dimensional mapping of temporo-limbic regions and the lateral ventricles in schizophrenia: gender effects. Biol Psychiatry 50:84-97. doi:10.1016/S0006-3223(00)01120-3

52. Newton SS, Duman RS (2007) Neurogenic actions of atypical antipsychotic drugs and therapeutic implications. CNS Drugs 21:715-725. doi:10.2165/00023210-200721090-00002

53. Pakkenberg B, Pelvig D, Marner L et al (2003) Aging and the human neocortex. Exp Gerontol 38:95-99. doi:10.1016/S0531-55 65(02)00151-1

54. Pegues MP, Rogers LJ, Amend D et al (2003) Anterior hippocampal volume reduction in male patients with schizophrenia. Schizophr Res 60:105-115. doi:10.1016/S0920-9964(02)00288-8

55. Price JL, Ko AI, Wade MJ et al (2001) Neuron number in the entorhinal cortex and CA1 in preclinical Alzheimer disease. Arch Neurol 58:1395-1402. doi:10.1001/archneur.58.9.1395

56. Rajarethinam R, DeQuardo JR, Miedler J et al (2001) Hippocampus and amygdala in schizophrenia: assessment of the relationship of neuroanatomy to psychopathology. Psychiatry Res 108:79-87. doi:10.1016/S0925-4927(01)00120-2

57. Rametti G, Segarra N, Junque C et al (2007) Left posterior hippocampal density reduction using VBM and stereological MRI procedures in schizophrenia. Schizophr Res 96:62-71. doi:10.1016/ j.schres.2007.04.034

58. Schmitz C (1998) Variation of fractionator estimates and its prediction. Anat Embryol (Berl) 198:371-397. doi:10.1007/ s004290050191

59. Schmitz C, Hof PR (2005) Design-based stereology in neuroscience. Neuroscience 130:813-831. doi:10.1016/j.neuroscience.2004.08.050

60. Schmitz C, Hof PR (2000) Recommendations for straightforward and rigorous methods of counting neurons based on a computer simulation approach. J Chem Neuroanat 20:93-114. doi:10.1016/ S0891-0618(00)00066-1

61. Segal D, Koschnick JR, Slegers LH et al (2007) Oligodendrocyte pathophysiology: a new view of schizophrenia. Int J Neuropsychopharmacol 10:503-511. doi:10.1017/S146114570600722X

62. Selemon LD, Mrzljak J, Kleinman JE et al (2003) Regional specificity in the neuropathologic substrates of schizophrenia: a morphometric analysis of Broca's area 44 and area 9. Arch Gen Psychiatry 60:69-77

63. Stark AK, Toft MH, Pakkenberg $\mathrm{H}$ et al (2007) The effect of age and gender on the volume and size distribution of neocortical neurons. Neuroscience 150:121-130. doi:10.1016/j.neuroscience.2007.06.062

64. Stark AK, Uylings HB, Sanz-Arigita E et al (2004) Glial cell loss in the anterior cingulate cortex, a subregion of the prefrontal cortex, in subjects with schizophrenia. Am J Psychiatry 161:882-888. doi:10.1176/appi.ajp.161.5.882
65. Steiner J, Bernstein HG, Bielau H et al (2008) S100B-immunopositive glia is elevated in paranoid as compared to residual schizophrenia: a morphometric study. J Psychiatr Res 42:868-876. doi:10.1016/j.jpsychires.2007.10.001

66. Sterio DC (1984) The unbiased estimation of number and sizes of arbitrary particles using the disector. J Microsc 134:127-136

67. Szeszko PR, Goldberg E, Gunduz-Bruce H et al (2003) Smaller anterior hippocampal formation volume in antipsychotic-naive patients with first-episode schizophrenia. Am J Psychiatry 160:2190-2197. doi:10.1176/appi.ajp.160.12.2190

68. Tkachev D, Mimmack ML, Ryan MM et al (2003) Oligodendrocyte dysfunction in schizophrenia and bipolar disorder. Lancet 362:798-805. doi:10.1016/S0140-6736(03)14289-4

69. Tregellas JR, Tanabe JL, Miller DE et al (2004) Neurobiology of smooth pursuit eye movement deficits in schizophrenia: an fMRI study. Am J Psychiatry 161:315-321. doi:10.1176/appi.ajp. 161.2.315

70. Uranova NA, Vostrikov VM, Orlovskaya DD et al (2004) Oligodendroglial density in the prefrontal cortex in schizophrenia and mood disorders: a study from the Stanley Neuropathology Consortium. Schizophr Res 67:269-275. doi:10.1016/S0920-9964(03) 00181-6

71. Uranova NA, Vostrikov VM, Vikhreva OV et al (2007) The role of oligodendrocyte pathology in schizophrenia. Int J Neuropsychopharmacol 10:537-545. doi:10.1017/S1461145707007626

72. van Kooten IA, Palmen SJ, von Cappeln P et al (2008) Neurons in the fusiform gyrus are fewer and smaller in autism. Brain 131:987-999. doi:10.1093/brain/awn033

73. Velakoulis D, Stuart GW, Wood SJ et al (2001) Selective bilateral hippocampal volume loss in chronic schizophrenia. Biol Psychiatry 50:531-539. doi:10.1016/S0006-3223(01)01121-0

74. Vostrikov V, Orlovskaya D, Uranova N (2008) Deficit of pericapillary oligodendrocytes in the prefrontal cortex in schizophrenia. World J Biol Psychiatry 9:34-42. doi:10.1080/15622970701 210247

75. Walker MA, Highley JR, Esiri MM et al (2002) Estimated neuronal populations and volumes of the hippocampus and its subfields in schizophrenia. Am J Psychiatry 159:821-828. doi:10.1176/appi.ajp.159.5.821

76. West MJ, Coleman PD, Flood DG et al (1994) Differences in the pattern of hippocampal neuronal loss in normal ageing and Alzheimer's disease. Lancet 344:769-772. doi:10.1016/S0140-6736(94) 92338-8

77. West MJ, Slomianka L, Gundersen HJ (1991) Unbiased stereological estimation of the total number of neurons in the subdivisions of the rat hippocampus using the optical fractionator. Anat Rec 231:482-497. doi:10.1002/ar.1092310411

78. White T, Kendi AT, Lehericy S et al (2007) Disruption of hippocampal connectivity in children and adolescents with schizophrenia-a voxel-based diffusion tensor imaging study. Schizophr Res 90:302-307. doi:10.1016/j.schres.2006.09.032

79. Williams RW, Rakic P (1988) Three-dimensional counting: an accurate and direct method to estimate numbers of cells in sectioned material. J Comp Neurol 278:344-352. doi:10.1002/ cne. 902780305

80. Wright IC, Rabe-Hesketh S, Woodruff PW et al (2000) Meta-analysis of regional brain volumes in schizophrenia. Am J Psychiatry 157:16-25

81. Yakovlev PI, Lecours AR (1967) The myelogenetic cycles of regional maturation of the brain. Blackwell Scientific Publications, Oxford, pp 3-70

82. Yamasue H, Iwanami A, Hirayasu Y et al (2004) Localized volume reduction in prefrontal, temporolimbic, and paralimbic regions in schizophrenia: an MRI parcellation study. Psychiatry Res 131:195-207. doi:10.1016/j.pscychresns.2004.05.004 
83. Zaidel DW, Esiri MM, Harrison PJ (1997) Size, shape, and orientation of neurons in the left and right hippocampus: investigation of normal asymmetries and alterations in schizophrenia. Am J Psychiatry 154:812-818

84. Zhang Z, Sun J, Reynolds GP (2002) A selective reduction in the relative density of parvalbumin-immunoreactive neurons in the hippocampus in schizophrenia patients. Chin Med J 115:819-823
85. Zhou Y, Shu N, Liu Y et al (2008) Altered resting-state functional connectivity and anatomical connectivity of hippocampus in schizophrenia. Schizophr Res 100:120-132. doi:10.1016/j.schres. 2007.11.039 